\title{
Late Pleistocene vegetation from the Thimi Formation, Kathmandu Valley, Nepal
}

Khum N Paudayal

Central Department of Geology, Tribhuvan University, Kirtipur, Kathmandu, NEPAL

For correspondence, E-mail: knpaudayal@wlink.com.np

The Thimi Formation is characterized by alternation of fine to medium-grained sand, silt, siltyclayand clay, deposited by distal fluvial system. Radiocarbon ages of two wood samples from middlepart of thesection (13m from thetop) and from the basal part (21 $\mathrm{m}$ from the top) are found to be $41,700 \pm \frac{5600}{3200}$ and $>37,900$ yrs BP, respectively. Altogether 40 samples were taken

FIGURE 1. Zonation of vegetation in the Central Midlands of Nepal ( $\mathrm{K}=\mathrm{Kathmandu}$ )

\section{S}

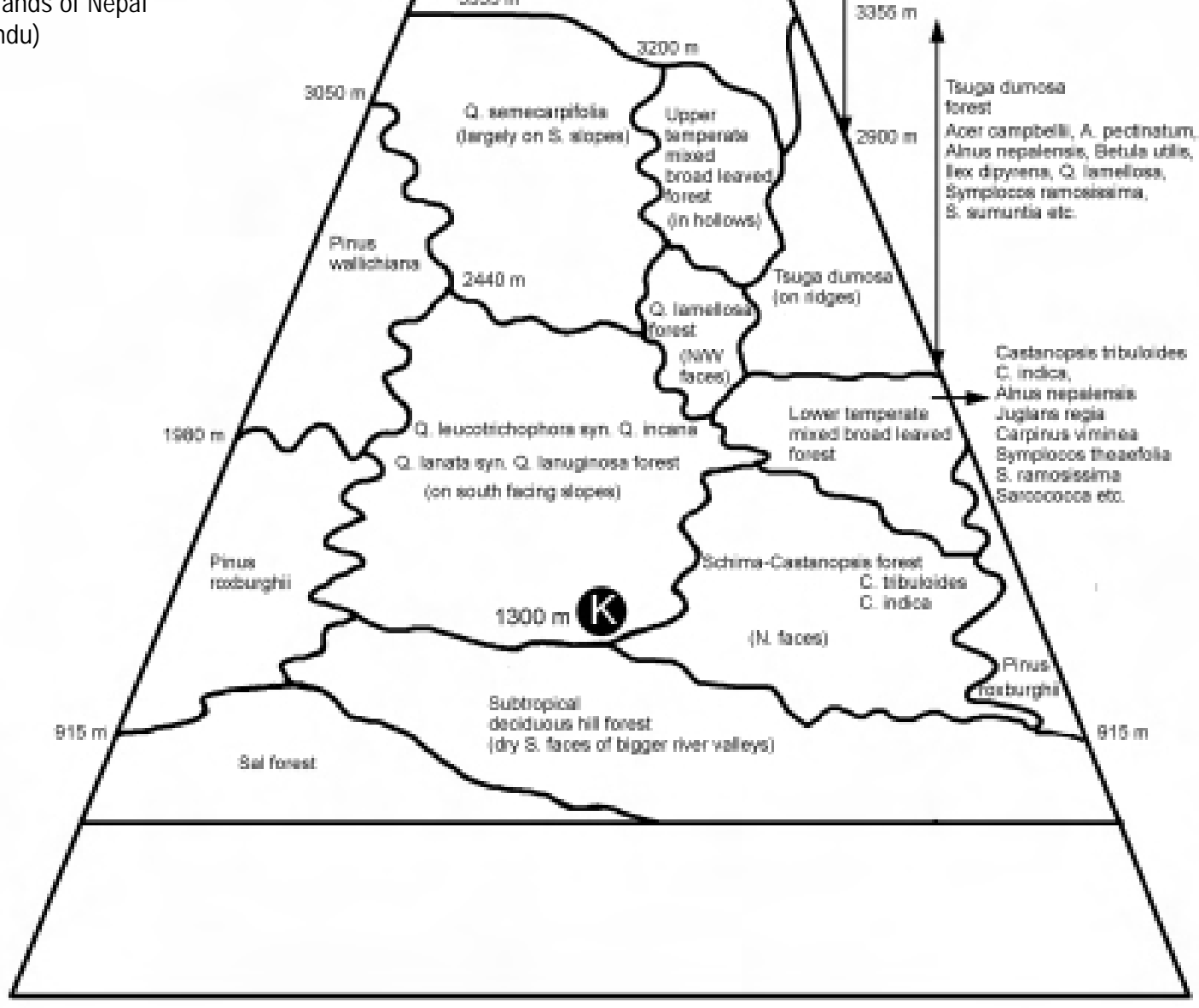


from $23 \mathrm{~m}$ thick type section of the Thimi Formation for palynological study. The pollen display only minor fluctuations in their assemblages without having any major break (Figures 1 and2). On the basis of theseminor changesthissection is divided into seven pollen zones. Thesmall-scalechangesfrom onepollen zone to another can be explained as "cold", "fairly cold" or "moderately warm" climatic conditions. For example the presence of Castanopsis together with high amounts of Quercus and small amounts of Abies and Picea can be assigned to a moderately warm climate. Thisisfollowed byan increasein Pinus, Picea, Abiesand Tsuga along with thecontemporaneous decrease of Castanopsisand Quercusindicatinga colder climate. In general the pollen assemblages of the Thimi Formation are dominated by cold elementssuch asPinuswallichiana and differentQuercus species that grow in the warm to cool temperate zone of Nepal. This is confirmed by high percentages of Poaceae and other herbaceous plants with very little woody angiosperms. Gymnosperms such as Pinus wallichiana (along with Abies spectabilis, Tsuga dumosa and Picea smithiana) were the dominant over the woody angiosperms (Quercus lanata, Q. lamellosa and Q. leucotrichophora and Q. semecarpifolia). An attempt was made to quantify the climatic change by plotting the present altitudinal ranges of all available pollen taxa from a single horizon. It would appear that the vegetation in the Kathmandu Valley shifted to a lower altitude by approximately 1000 m during the latePleistocene. If weallow for a temperature lapse rate of $0.6{ }^{\circ} \mathrm{C} / 100 \mathrm{~m}$ in the Nepal Himalayas this must indicate a lowering of the temperature by 6 to $7^{\circ} \mathrm{C}$.

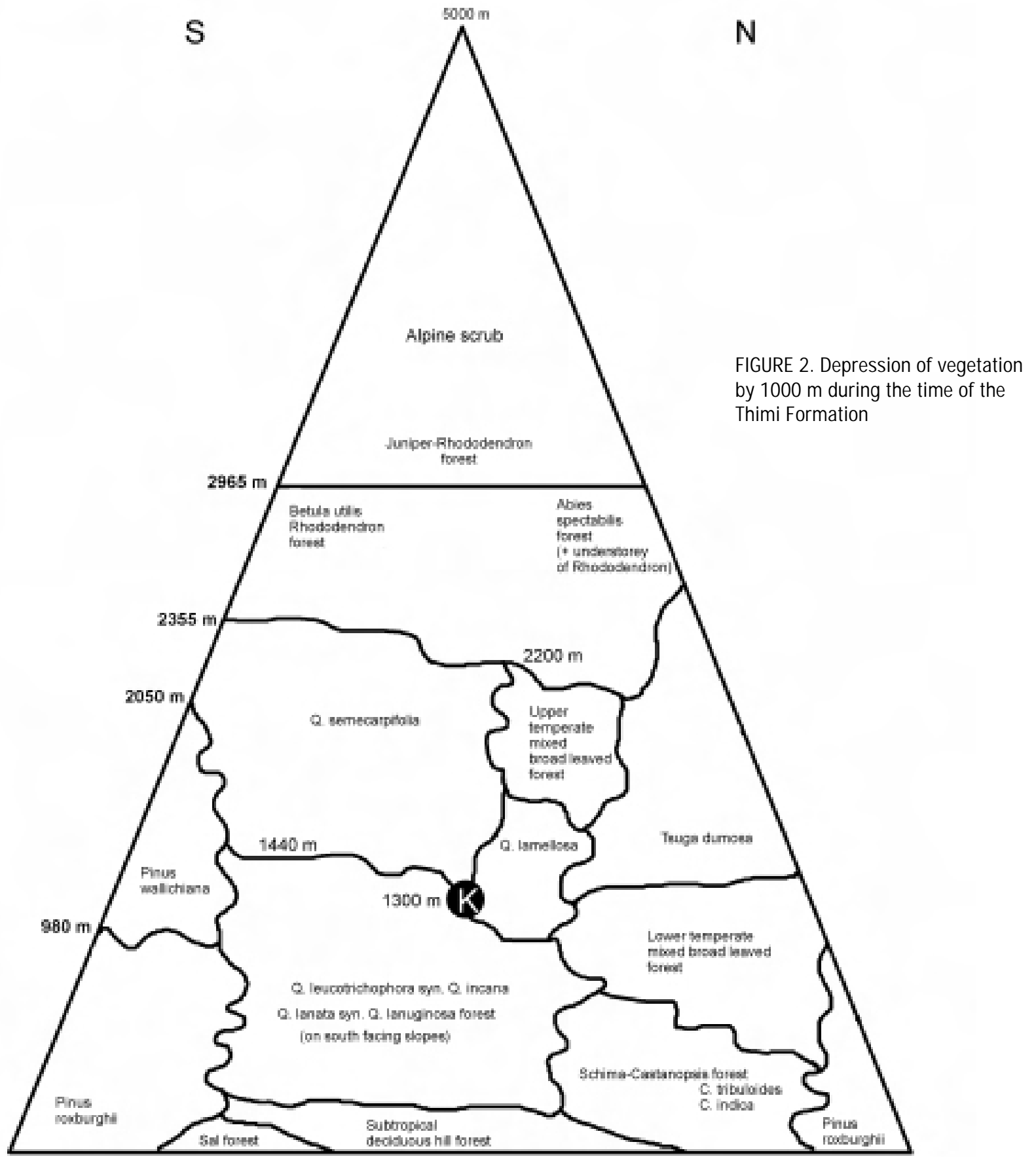

